\title{
Design of entertainment mobile robot: IDAP
}

\begin{abstract}
Robotic system applications have advanced dramatically over the past few years. This paper presents the IDAP Robot, which is designed and fabricated by taking the first Malaysia robot games festival or Robofest 2002. Development of the IDAP Robot based on the contest regulation to place out the beach balls into the cylinder tubes. This paper focuses on strategies motion of the IDAP robot. The strategies that involve are; it can carry out eighteen beach balls in one time and it is able to place all the cylinder tubes in one track within 3 minutes. The structure design of the robot is divided into two parts namely hardware and software parts. The hardware part involves the design and development of the platform module, storage module, arm manipulator module, power supply module, permanent magnet DC motor, sensing system, control panel, circuit protection and programmable logic controller (PLC). The platform module is divided into three parts namely, Y-axis design, Z-axis design and ofaxis design.
\end{abstract}

Keyword: Automation; Cylindrical coordinate approach; Mobile robot 